\title{
A traffic-stopping prediction tool?
}

An update to a free tool to aid men and their doctors in assessing their risk of prostate cancer has been released by the University of Texas Science Health Center, San Antonio. The original calculator was developed in 2006 by Ian Thompson and colleagues, and was based on data collected from 5,519 men involved in the placebo arm of the Prostate Cancer Prevention Trial (PCPT).

The tool predicts the probability of receiving one of three possible biopsy results-no cancer, low-grade cancer or high-grade cancer-using individual patient information, including PSA level, family history of prostate cancer, age, ethnicity and rectal examination findings. The results provided by the calculator then assist the patient and their doctor in choosing treatment options, weighing the risks and the benefits of biopsy against the likelihood of a cancer diagnosis.

The previous incarnation of the calculator had been validated in several patient populations, but the new version now contains data from the 18,882 participants in the PCPT as well as the San Antonio Center of Biomarkers of Risk for Prostate Cancer trial. With these additions, it is hoped that the tool will be more robust than version 1.0. More-advanced statistical modelling has also been used to distinguish the likelihood of finding low-grade or high-grade disease.

The upgraded calculator takes 3 min to complete and gives patients more information about their risk of having high-grade or low-grade cancer than the previous tool. The output gives the calculated percentage chance of biopsy result and also incorporates a new, user-friendly 'emoji' graphic to represent these calculated risks pictorially: with a sad, red face depicting high-grade cancer risk; a neutral, yellow face for low-grade risk and a happy, green face for a negative biopsy. The tool also takes into account that $2-4 \%$ of men undergoing biopsy get an infection that might require hospitalization.

This tool aims to help in the treatment decision-making process for doctors and their patients, and also predict the chance of detecting highrisk cancers against finding low-grade cancer, which might not require treatment, or no cancer at all. Patients with a low percentage chance of having high-grade cancer might choose to defer treatment in favour of active surveillance. Alternatively, they might choose watchful waiting and, therefore, not risk the adverse effects that are associated with the biopsy procedure, the reduction in quality of life and anxiety that can result from a diagnosis of low-risk cancer.

Future developments in the prediction calculator might include analysis of other factors, such as comorbidities, but this updated version should further assist the treatment decision-making process.

Louise Stone
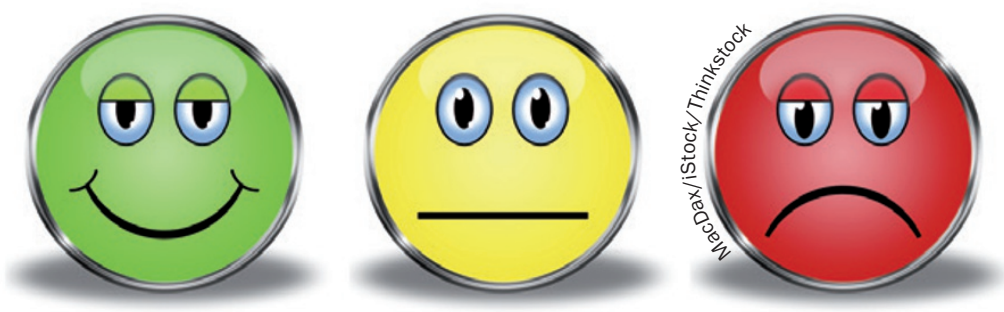

Original article Thompson, I. M. et al. Focusing PSA testing on detection of high-risk prostate cancers by incorporating patient preferences into decision making. JAMA doi:10.1001/jama.2014.9680

Further reading Ankerst, D. P. Prostate Cancer Prevention Trial Risk Calculator 2.0 for the prediction of low- vs high-grade prostate cancer. Urology 83, 1362-1368 (2014) 